\title{
Inestabilidad de la articulación tibio- peronea proximal como causa de dolor en artroplastia total de rodilla primaria.
}

DOI: http//dx.doi.org/10.37315/SOTOCAV202028355114

RODRÍGUEZ COLLELL JR ${ }^{1}$, MIFSUT MIEDES $D^{1,2}$.

1. DEPARTAMENTO DE SALUD CLÍNICO-MALVARROSA DE VALENCIA

2. DEPARTAMENTO DE CIRUGÍA UNIVERSIDAD DE VALENCIA

\begin{abstract}
Resumen.
La inestabilidad de la articulación tibio-peronea proximal (ATPP) aislada como causa de dolor en una rodilla protetizada es una entidad clínica muy poco frecuente, hasta la fecha solo hemos encontrado una referencia en la bibliografía de inestabilidad ATPP asociada a Artroplastia total de rodilla. Revisamos la bibliografía sobre las diferentes formas de inestabilidad y su etiología, así como las posibilidades de tratamiento conservador y quirúrgico. Presentamos el caso de un paciente con inestabilidad ATPP anterolateral asociada a una ATR primaria posteroestabilizada (PS) con platillo tibial móvil.
\end{abstract}

Palabras clave: Inestabilidad tibioperonea proximal, artroplastia rodilla dolorosa, artrodesis.

\section{Summary.}

The isolated instability of the proximal tibiofibular joint (PTFJ) as a cause of pain in a prosthetic knee is a very rare clinical entity, to date we have only found one reference in the bibliography of ATPP instability associated with total knee arthroplasty. We review the bibliography on the different forms of instability and their etiology, as well as the possibilities of conservative and surgical treatment. We present the case of a patient with anterolateral instability PTFJ associated with a primary postero-stabilized (PS) TKA with a mobile tibial polyethylene.

\section{Correspondencia:}

Juan Ramón Rodríguez Collell

rocojura@hotmail.com

\section{Introducción}

La inestabilidad de la articulación tibio-peronea proximal aislada es una entidad clínica poco frecuente, generalmente asociada a traumatismos deportivos, aunque a veces, bajo ciertas condiciones patológicas, puede existir cierta predisposición a la luxación de la cabeza del peroné.

La lesión de la articulación tibioperonea proximal (ATPP) se observa con mayor frecuencia entre los atletas cuyos deportes requieren movimientos violentos de torsión de la rodilla flexionada, como fútbol, rugby, béisbol y baloncesto ${ }^{1-6}$, y se suelen clasificar en 6 tipos: subluxación, luxación anterolateral, posteromedial, superior, posterolateral $\mathrm{e}^{\text {inferior } 3,7,8}$. De estos, la luxación anterolateral es la más común $(69 \% \text { al } 85 \% \text { de los casos) })^{9,10}$. Las 2 últimas luxaciones (posterolateral e inferior) son muy inusuales y se asocian a rodilla flotante. Sus diagnósticos se basan principalmente en la presencia de lesiones neurovasculares. Las opciones de tratamiento para las luxaciones de la ATPP van desde la reducción cerrada a la reducción abierta con reconstrucción ligamentosa, resección de la cabeza del peroné y artrodesis ${ }^{7,8}$. Hasta la fecha solo hemos encontrado una referencia en la bibliografía de inestabilidad ATPP asociada a Artroplastia total de rodilla ${ }^{9}$. Presentamos el caso de un paciente con inestabilidad ATPP anterolateral asociada a una ATR primaria posteroestabilizada (PS) con platillo tibial móvil.

\section{Caso Clínico}

Mujer de 68 años de edad. Sin antecedentes de interés, que fue intervenida en el año 2013 por gonartrosis severa femorotibial medial en la rodilla derecha, implantándose una prótesis de rodilla primaria "Attune" (Depuy $®)$ posteroestabilizada de platillo móvil.

La evolución de la paciente fue buena con un rango de movimiento $0-120^{\circ}$ y una rodilla estable e indolora. Sin embargo, a los dos años de la cirugía la paciente empezó con un cuadro de dolor intenso en la rodilla localizado a nivel femorotibial lateral, sin antecedente traumático. El dolor era muy intenso y 
no mejoraba con analgésicos, antiinflamatorios $u$ opiodes. La Rx simple no mostraba signos de aflojamiento o de infección (Fig. 1). La analítica sanguínea era normal con cifras de leucocitos, PCR y VSG normales.

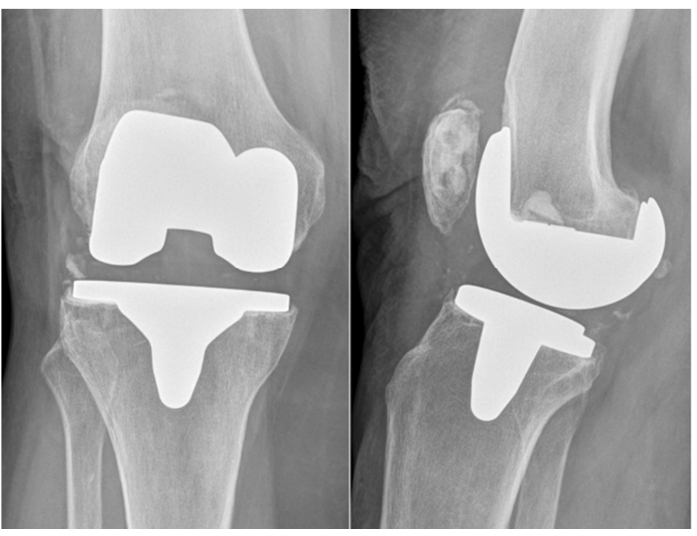

Figura 1: Imagen de radiografía simple de la rodilla intervenida.

Se realizó una gammagrafía ósea con administración iv de $29,2 \mathrm{mCi}$ de $99 \mathrm{~m}$ Tc-HMDP. El estudio con radioisótopos mostró en la fase vascular inicial y en las fases estática precoz y tardía una hipercaptación del radiotrazador en la articulación tibioperonea proximal que podría corresponder a un proceso degenerativo osteoarticular, sin objetivar hipercaptación periprotésica que sugiriera aflojamiento protésico ( Fig. 2).

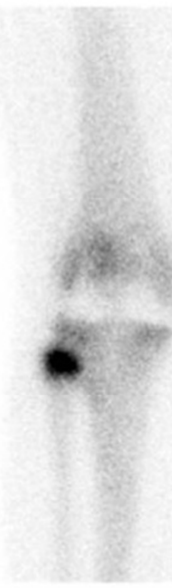

Figura 2: Imagen de gammagrafía ósea donde se observa la hipercaptación del radiotrazador en la articulación tibioperonea proximal.

Motivo por el que solicitamos una TAC que mostró una disminución del espacio articular de la articulación tibioperonea proximal sin otras alteraciones (Fig. 3).

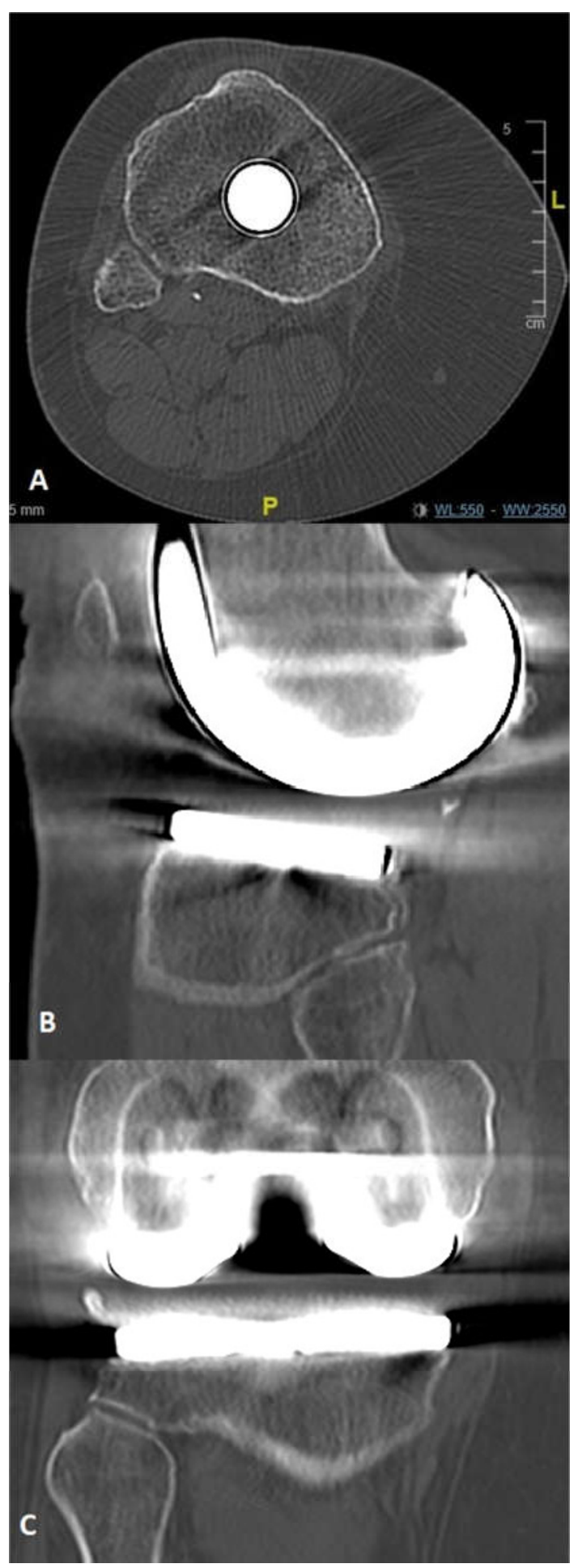

Figura 3: Cortes de TAC (A: axial, B: sagital y C: coronal) donde se observa la ATPP con signos degenerativos.

Sospechando que la causa del dolor era una inestabilidad y una artrosis de la articulación tibioperonea proximal, infiltramos la sindesmosis tibioperonea proximal con un vial de $2 \mathrm{ml}$ celestone cronodose (22,8 mg de Betametasona) y $2 \mathrm{ml}$ de SCANDINIBSA $20 \mathrm{mg} / \mathrm{ml}+0,01 \mathrm{mg} / \mathrm{ml}$ Solución inyectable (Mepivacaína Hidrocloruro $20,0 \mathrm{mg} / \mathrm{ml}$ y 
Epinefrina tartrato $0,018 \mathrm{mg} / \mathrm{ml}) ;$ sin embargo, la clínica dolorosa no mejoró con la infiltración. A pesar de ello, mantuvimos el diagnóstico de inestabilidad y artrosis de la articulación tibioperonea proximal como causa del dolor por lo que se decidió intervenir a la paciente en febrero del año 2019.

Se realizó una incisión transversal sobre la articulación tibioperonea proximal. Se desinsertó la musculatura del compartimento tibial anterior de sus inserciones proximales y anteriores de la tibia, exponiendo la sindesmosis tibioperonea proximal. Se identificó el nervio peroneo común sobre el cuello del peroné para evitar su lesión. Se observó una inestabilidad franca de la articulación tibioperonea que estaba hipermóvil, con una destrucción total de cualquier tipo de unión entre el peroné y la tibia. Se cruentó la superficie articular del peroné y de la tibia, y se aportó injerto de hueso esponjoso de la cresta iliaca ipsilateral. La articulación una vez reducida se fijó con un tornillo de esponjosa de rosca parcial de $4,5 \mathrm{~mm}$ con arandela.

La paciente se dejó en descarga durante 6 semanas, tras las cuales empezó la deambulación con carga parcial asistida con muletas.

La evolución de la paciente fue satisfactoria con desaparición completa del dolor, conservando una movilidad de $0-120^{\circ}$, una rodilla estable y una deambulación sin muletas. Se muestra el último control radiográfico con la artrodesis tibioperonea proximal (Fig. 4).

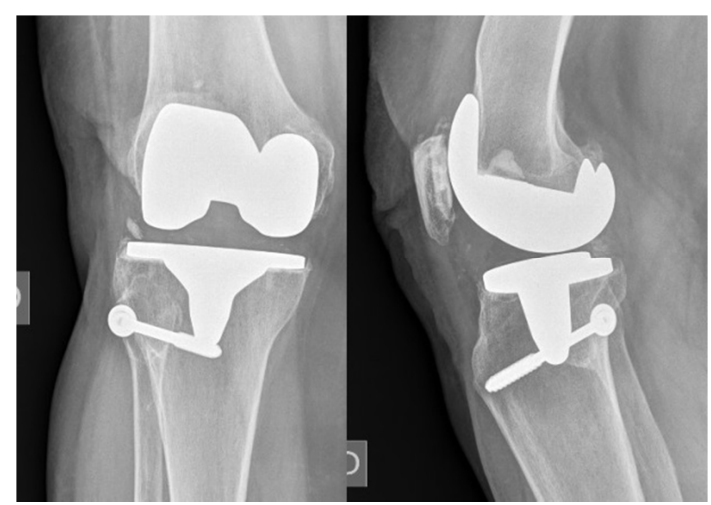

Figura 4: Imagen de radiografía simple donde se muestra la artrodesis de la ATPP realizada.

\section{Discusión}

La articulación tibio-peronea proximal es una articulación de cartílago hialino con dos superficies articulares, revestida con una membrana sinovial y cápsula fibrosa, que se comunica con la articulación de la rodilla en el $10 \%$ al $12 \%$ de personas ${ }^{10,11}$. Su estabilidad viene determinada por una estructura ligamentosa consistente. Anteriormente presenta el fuerte ligamento tibio-peroneo anterior, que se compone de tres bandas anchas y una extensión del tendón del bíceps. $\mathrm{Y}$ las estructuras posteriores aúnan al relativamente débil ligamento tibio-peroneo posterior con una sola banda, el tendón poplíteo y el ligamento poplíteo-peroneo. Superiormente se inserta el ligamento colateral peroneo, que es el principal estabilizador de esta articulación (su laxitud puede favorecer la luxación o subluxación) $)^{12,13}$. Ogden ${ }^{14}$ evaluó el movimiento anteroposterior del peroné proximal con cambios de posición de la rodilla. Con flexión de rodilla, el peroné se desplaza anteriormente, mientras que en la extensión lo hace posteriormente. Según Ogden, en la mayoría de los pacientes, con la rodilla flexionada, la cabeza del peroné podría moverse aproximadamente $1 \mathrm{~cm}$ en ambas direcciones, anterior y posterior, mientras que con la rodilla extendida, la excursión de la cabeza del peroné es mínima. También hay un ligero movimiento hacia arriba del peroné debido a expansión transversal forzada de la mortaja maleolar durante la dorsiflexión máxima del tobillo ${ }^{15}$. La anatomía de la ATPP está indudablemente relacionada directamente con su estabilidad funcional, ya que puede soportar tensiones aplicadas de forma longitudinal o axial. Aproximadamente una sexta parte de la carga aplicada en el tobillo se transmite a lo largo del peroné hasta la ATPP, y el peroné proximal está involucrado en fuerzas de tracción ${ }^{16,17}$.

\section{Las funciones principales de la ATPP son:}

(1) disipación de tensiones torsionales aplicadas en el tobillo,

(2) disipación de los momentos de fuerza en flexión tibial lateral, $y$

(3) soporte de peso en tracción, en lugar de compresión $^{14}$.

Existen variantes anatómicas de la ATPP que pueden clasificarse en 3 tipos:

El tipo I incluye ATPP con un superficie articular casi horizontal ( $<30$ de inclinación) y un área de superficie $>20 \mathrm{~mm}^{2}$.

Tipo II incluye ATPP con una gran superficie elíptica, cóncava en el peroné, y con frecuencia con comunicación con la rodilla.

Tipo III incluye ATPP con una superficie articular pequeña $(<15 \mathrm{~mm} 2)$ y una pendiente inclinación (> $30)^{18}$, con la que se correspondía nuestro caso. Estas variaciones anatómicas deben ser consideradas al tratar pacientes con inestabilidad de la ATPP.

En cuanto a la etiología de la inestabilidad en el caso que presentamos, no pudo determinarse si se produjo algún gesto durante la cirugía con los separadores que afectara a la estabilidad de la 
ATPP, poco probable, ya que la paciente estuvo asintomática durante dos años, o que el hecho de llevar un implante de polietileno móvil hubiese influido en crear dicha inestabilidad, aunque tampoco se ha podido confirmar.

Clínicamente, el dolor y la inestabilidad lateral de la rodilla se asocian a un marcado deterioro funcional $^{7,17}$. A menudo se diagnostica incorrectamente como otras lesiones mejor descritas y reconocidas en la parte lateral de la rodilla. Turco y Spinella6 describieron tres signos cardinales para el reconocimiento de la luxación anterolateral: 1) desplazamiento lateral del peroné en radiografía anteroposterior; 2) aumento del espacio interóseo proximal, y 3) desplazamiento anterior de la cabeza del peroné en la radiografía lateral. En nuestro caso no había desplazamiento articular pero sí inestabilidad. El diagnóstico precoz basado en la exploración física, estudios con radiología simple y, en caso de dudas, confirmado con TAC ${ }^{12}$ permiten un buen pronóstico. En nuestro caso fue de gran ayuda la gammagrafía, ya que el depósito del radiotrazador se localizaba de forma significativa en dicha articulación. En las inestabilidades crónicas la RMN también puede aportar información.

Hay varias opciones de tratamiento para la inestabilidad de la ATPP, la mayoría destinadas a corregirla mientras se conserva su movimiento. En el caso de luxación aguda, se suele realizar reducción cerrada, con o sin inmovilización, con la rodilla en extensión o leve flexión durante 2-3 semanas ${ }^{3}$; aunque a veces se requiere reducción abierta y fijación como describe Anderson18 con agujas de Kirschner. Es controvertido si la carga de la extremidad debe realizarse después del procedimiento $^{19}$. Se requiere estabilización quirúrgica hasta en un $57 \%$ de los casos tardíos o recurrentes, debido al dolor persistente e inestabilidad crónica ${ }^{3,17}$. La reducción abierta puede ser necesaria en los pacientes que experimentan lesiones crónicas, reducción cerrada fallida, reducción inestable o lesiones neurovasculares con fracturas asociadas. Entre las opciones quirúrgicas para el tratamiento de dicha inestabilidad crónica se incluyen la resección de la cabeza del peroné, artrodesis de la ATPP con o sin osteotomía del peroné, reducción abierta y fijación interna con o sin extracción de material, y la reconstrucción de ligamentos utilizando la banda iliotibial o el tendón del bíceps femoral (ligamentoplastia de Weinert ${ }^{20}$ y Giachino $^{21}$ con hemitendón del bíceps femoral $)^{3,22-}$ ${ }^{24}$. Actualmente no existe ninguna técnica o informe publicado disponible para guiar el enfoque del tratamiento para este tipo de paciente, por lo tanto, en nuestro caso, dado el deterioro articular observado y la inestabilidad manifiesta, se optó por realizar una artrodesis.

La decisión de retirar el material después de la artrodesis sigue siendo controvertida. Van den Bekerom y cols. ${ }^{25}$ realizó artrodesis de la ATPP con extracción temprana de tornillos a los 3 a 6 meses y obtuvo buenos resultados en deportistas. Aunque se encuentran disponibles múltiples procedimientos quirúrgicos, actualmente no hay evidencia clara que respalde el uso de uno sobre el otro. La osteotomía del peroné en el momento de la artrodesis ATPP no suele realizarse, incluso si los estudios muestran un riesgo de posible desarrollo tardío de dolor de tobillo, debido a la pérdida de la rotación normal del peroné $^{14}$, aunque es una opción si se manifiesta dolor de tobillo tardío.

Las opciones de tratamiento quirúrgico para la ATPP no están exentas de complicaciones, como la parálisis del nervio peroneo ( $3 \%-5 \%)$, artritis, infecciones, inestabilidad crónica, artritis de tobillo, dolor crónico y trombosis venosa profunda ${ }^{3,9,17}$. 
RODRÍGUEZ-COLLELL JR Y MIFSUT-MIEDES D. Inestabilidad de la articulación tibio-peronea proximal como causa de dolor en artroplastia total de rodilla.

\section{Bibliografía}

1. Lord CD, Coutts JW. A study of typical parachute injuries occurring in two hundred and fifty thousand jumps at the parachute school. J Bone Joint Surg 1944; 26:547.

2. Ogden JA. Dislocation of the proximal fibula. Radiology 1972; 105(3):547.

3. Ogden JA. Subluxation and dislocation of the proximal tibiofibular joint. J Bone Joint Surg Am 1974; 56(1):145.

4. Shapiro GS, Fanton GS, Dillingham MF. Reconstruction for recurrent dislocation of the proximal tibiofibular joint. A new technique. Orthop Rev 1993; 22(11):1229.

5. Thomason PA, Linson MA. Isolated dislocation of the proximal tibiofibular joint. J Trauma 1986; 26(2):192.

6. Turco VJ, Spinella AJ. Anterolateral dislocation of the head of the fibula in sports. Am J Sports Med 1985; 13(4):209.

7. Semonian RH, Denlinger PM, Duggan RJ. Proximal tibiofibular subluxation relationship to lateral knee pain: a review of proximal tibiofibular joint pathologies. J Orthop Sports Phys Ther 1995; 21(5):248.

8 Sreesobh KV, Cherian J. Traumatic dislocations of the proximal tibiofibular joint: a report of two cases. J Orthop Surg (Hong Kong) 2009; 17(1):109.

9. Bedard M, Corriveau-Durand S. Instability of the proximal tibiofibular joint associated with total knee arthroplasty. Arthroplasty Today 2016; 2:93-6.

10. Eichenblat M, Nathan H. The proximal tibiofibular joint. An anatomical study with clinical and pathological considerations. Int Orthop 1983; $7(1): 31$.

11. Resnick D, Newell JD, Guerra Jr J, et al. Proximal tibiofibular joint: anatomicpathologic-radiographic correlation. Am J Roentgenol 1978; 131(1):133

12. Voglino JA, Denton JR. Acute traumatic proximal tibiofibular joint dislocation confirmed by computed tomography. Orthopedics 1999 ; 22(2):255-8.

13. Espregueira-Mendes JD, da Silva MV. Anatomy of the proximal tibiofibular joint. Knee Surg Sports Traumatol Arthrosc 2006; $14(3): 241$.

14. Ogden JA. The anatomy and function of the proximal tibiofibular joint. Clin Orthop Relat Res 1974; 101:186.

14 Eichenblat M, Nathan $\mathbf{H}$. The proximal tibiofibular joint. An anatomical study with clinical and pathological considerations. Int Orthop 1983; 7(1):31.

15. Moore KL, Dalley AF, Agur AM, Limb L. Clinically oriented anatomy. 6th ed. New Delhi: Lippincott and Williams and Wilkins, Wolters Kluwer India Pvt; 2010. p. 508.

16. Lambert KL. The weight-bearing function of the fibula. A strain gauge study. J Bone Joint Surg Am 1971; 53(3):507.

17. Preuschoft H. [Mechanical stress on the fibula of primates]. Gegenbaurs Morphol Jahrb 1971; 117(2):211.

16. Barnett $\mathbf{C H}$, Napier JR. The axis of rotation at the ankle joint in man; its influence upon the form of the talus and the mobility of the fibula. J Anat 1952; 86(1):1.

17. Sekiya JK, Kuhn JE. Instability of the proximal tibiofibular joint. J Am Acad Orthop Surg 2003; 11(2):120.

18. Anderson K. Dislocation of the superior tibiofibular joint. Injury 1985; 16:494-8.

19. Burke NG, Robinson E, Thompson NW. An isolated proximal tibiofibular joint dislocation in a young male playing soccer: a case report. Cases J 2009; 2:7261.

20. Weinert CR, Raczka R. Recurrent dislocations of the superior tibiofibular joint, surgical stabilization by ligament reconstruction. J Bone Joint Surg Am 1986; 68:126-8.

21. Giachinno AA. Recurrent dislocations of the proximal tibiofibular joint. J Bone Joint Surg Am 1986; 68:1104-6.

22. Cazeneuve JF, Bracq H, Meeseman M. Weinert and Giachino ligament arthroplasty for the surgical treatment of chronic superior tibiofibular joint instability. Knee Surg Sports Traumatol Arthrosc 1997; 5(1):36.

23. Mena H, Brautigan B, Johnson DL. Split biceps femoris tendon reconstruction for proximal tibiofibular joint instability. Arthroscopy 2001; 17(6):668.

24. White SH. Bicipetal tenodesis for anterior subluxation of the superior tibiofibular joint. J Bone Joint Surg Br 2001; 83(8):1176.

25. Van den Bekerom MP, Weir A, van der Flier RE. Anatomic reconstruction or temporary screw stabilization of chronic symptomatic anterolateral proximal tibiofibular joint instability. Knee Surg Sports Traumatol Arthrosc 2011; 19(8):1406. 